\title{
Consistency of vitamin and/or mineral supplement use and demographic, lifestyle and health-status predictors: findings from the European Prospective Investigation into Cancer and Nutrition (EPIC)-Heidelberg cohort
}

\author{
Kuanrong $\mathrm{Li}^{1}$, Rudolf Kaaks ${ }^{1}$, Jakob Linseisen ${ }^{1,2}$ and Sabine Rohrmann ${ }^{3 *}$ \\ ${ }^{1}$ Division of Cancer Epidemiology, German Cancer Research Centre, Heidelberg, Germany \\ ${ }^{2}$ Institute of Epidemiology, Helmholtz Centre Munich, Neuherberg, Germany \\ ${ }^{3}$ Institute of Social and Preventive Medicine, University of Zurich, Hirschengraben 84, 8001 Zurich, Switzerland \\ (Received 25 November 2009 - Revised 2 March 2010 - Accepted 1 April 2010 - First published online 5 May 2010)
}

Cross-sectional studies suggest that dietary supplement use is associated with favourable demographic and lifestyle factors and certain health conditions. However, factors that affect the consistency of supplement use have not been investigated in prospective cohort studies. The aim of the present study was to seek baseline demographic, lifestyle and health-status predictors of subsequent consistent vitamin and/or mineral supplement use. A total of 8968 men and 10672 women of the European Prospective Investigation into Cancer and Nutrition (EPIC)-Heidelberg cohort, who answered the supplement-use questions in the baseline survey and two follow-up surveys, were categorised into three groups: consistent, inconsistent and never users. At baseline, $28.5 \%$ of men and $38.6 \%$ of women reported vitamin and/or mineral supplement use. After a median followup of 8.5 years, $14.6 \%$ of men and $22.9 \%$ of women were consistent users. During follow-up, $36.0 \%$ of male and $26.6 \%$ of female initial users stopped supplement use, whereas $27.8 \%$ of male and $39.4 \%$ of female initial non-users started supplement use. Women were more likely to be consistent users than men. Older age ( $\geq 50$ years), lower BMI $\left(<25 \mathrm{~kg} / \mathrm{m}^{2}\right)$ and self-reported hyperlipidaemia were common predictors of consistent use for both sexes. Additional predictors included higher educational level for men, and being more physically active and higher lifetime alcohol consumption for women. Consistent users had the highest intake of dairy products, fish, fruits and vegetables, and wine but the lowest intake of total meat. We concluded that supplement use is a fairly unstable behaviour in free-living individuals. Individuals with a favourable lifestyle and healthier diet are more likely to show consistent supplementation.

Vitamins: Minerals: Supplements: Cohort studies: EPIC

Cross-sectional studies have shown that dietary supplement users, in contrast to non-users, are more likely to be women, more highly educated, more physically active, non-smokers, light alcohol drinkers, and to have a healthier diet ${ }^{(1-6)}$. As a result, these factors are usually handled as confounders in epidemiological studies that investigate the preventive effect of dietary supplements on chronic diseases. Studies have shown that dietary supplement users and non-users have different health-status characteristics ${ }^{(2,3,6,7)}$. On the other hand, the occurrence of some specific health conditions, such as hypertension and cancer, may increase or decrease supplement use $^{(2,8)}$. It is also important to note that dietary supplement use may be an unstable behaviour in free-living individuals. However, the consistency of dietary supplement use is unable to be clearly answered by cross-sectional studies, and so far has not been investigated in prospective studies. The main objective of the present study was to describe the consistency of vitamin and/or mineral supplement use (hereafter referred to simply as supplement use) and to seek demographic, lifestyle and health-status predictors of consistent supplement use in a prospective cohort study.

\section{Materials and methods \\ Study population and data collection}

The present study was based on the ongoing European Prospective Investigation into Cancer and Nutrition (EPIC)Heidelberg. The EPIC-Heidelberg cohort, as one of two Germen EPIC cohorts, was set up between 1994 and $1998^{(9)}$. The target population of EPIC-Heidelberg was the group of individuals who were aged 40-64 years (for men) or 35-64 years (for women) living in Heidelberg and surrounding areas. Detailed description of recruitment is available in a published paper ${ }^{(10)}$. In brief, a randomised sample of eligible subjects was obtained from the municipal registration offices. Each eligible subject was invited by an invitation letter and reminded by a telephone call in case of no response to the letter. Eventually, 11928 men and 13612 women joined the cohort, totally accounting for $38.3 \%$ of those initially invited. The present study was conducted according to the guidelines laid down in the Declaration of Helsinki and all procedures involving human subjects were approved by the Ethical Committee of Heidelberg

Abbreviation: EPIC, European Prospective Investigation into Cancer and Nutrition.

* Corresponding author: Dr Sabine Rohrmann, fax +41 44634 4909, email sabine.rohrmann@ifspm.uzh.ch 
Medical School. Written informed consent was obtained from all subjects.

All recruited subjects completed a validated selfadministered FFQ, in which the consumption of 148 food items in the previous 12 months was assessed. Baseline demographic and lifestyle information was collected in a selfadministered lifestyle questionnaire at recruitment. Consumption of alcoholic beverages (beer, wine, and spirits) at the ages of 20,30 and 40 years and at recruitment was estimated by asking subjects to specify the amount (in glasses) on each occasion and frequency (ranging from 'never' to 'five or more times a day'). Alcohol intake (g/d) was then derived from the above information using the German Food Code and Nutrient Data Base BLS II.3 (BgVV, Berlin, Germany). Average lifetime alcohol intake was defined as a mean of weighted alcohol intake at different ages and at recruitment, with weights equal to corresponding total subject-specific time under investigation. Height and weight at recruitment were measured in a physical examination. BMI was calculated by dividing body mass $(\mathrm{kg})$ by the square of height $\left(\mathrm{m}^{2}\right)$. Medical history was recorded in a computer-guided interactive interview.

Information on regular use of any dietary supplements was collected in the computer-guided interactive interview at baseline and two subsequent follow-up questionnaires, i.e. the second follow-up questionnaire (FUP2; 2001-3) and the third follow-up questionnaire (FUP3; 2004-6). At all three occasions, subjects were asked if they had regularly used any dietary supplements in the previous 4 weeks, with 'regularly' defined as daily use that endured for at least 1 week, or non-daily use of at least five doses with a fixed interval. Subjects who answered 'yes' were further required to name the supplements they had used. However, information on duration and dosage of supplement use was not collected.

All the subjects answered the supplement-use question in the interactive interview. By the end of the third follow-up survey, a total of 5900 subjects did not provide information on supplement use in at least one of the two follow-up surveys due to death before the second follow-up questionnaire or third follow-up questionnaire ( $n$ 973), loss to at least one of the two follow-up surveys ( $n$ 3528), or data missing ( $n$ 1399). These subjects were excluded from the present study. In total, 8968 men and 10672 women who answered the dietary supplement-use questions at all the three time points were left for analyses. We divided these subjects into three categories: consistent, inconsistent and never users, using the following definitions: (1) consistent users were defined as subjects reporting regular supplement use at all the three time points; (2) inconsistent users were defined as subjects reporting regular supplement use not at all the three time points; (3) never users were defined as subjects reporting no regular supplement use at all the three time points.

\section{Statistical methods}

We calculated the frequency of different supplement-use patterns and conducted the $\chi^{2}$ test to examine the difference between men and women. A multivariate multinomial logistic regression model was fitted to examine the association between the categories of supplement use and the baseline demographics (sex, age, and educational level), lifestyle factors (physical activity, smoking status, lifetime alcohol consumption) and health conditions at recruitment (selfreported prevalent hypertension, hyperlipidaemia, diabetes mellitus, cancer, and history of myocardial infarction or stroke), with never users as the reference category. All these factors were mutually adjusted for in the multivariate model. All the subjects were categorised into three age groups: $<50$ years, 50-59.9 years, and $\geq 60$ years. BMI was categorised into three groups: $<25 \mathrm{~kg} / \mathrm{m}^{2}, \quad 25-29.9 \mathrm{~kg} / \mathrm{m}^{2}$, and $\geq 30 \mathrm{~kg} / \mathrm{m}^{2}$. Lifetime alcohol intake was categorised into tertiles by using cut-off points derived separately for men and women $(13.31$ and $27.97 \mathrm{~g} / \mathrm{d}$ for men, and 2.54 and $7.40 \mathrm{~g} / \mathrm{d}$ for women). Non-alcohol drinkers were not considered separately because of the small proportion $(1.44 \%)$. Daily energy intake $(\mathrm{kJ} / \mathrm{d})$ was adjusted for body mass. Physical activity was categorised into four levels, i.e. inactive, moderately inactive, moderately active, and active, by using the Cambridge Physical Activity Index proposed by Wareham et $a l .{ }^{(11)}$. To compare the baseline diet across the three supplement-use categories, we performed the non-parametric Kruskal-Wallis test given the non-normal distribution of dietary variables. We also used the Bonferroni-Dunn test to conduct multiple comparisons if the omnibus difference was statistically significant. A two-sided $P<0.05$ was considered statistically significant. SAS 9.1 software (SAS Institute Inc., Cary, NC, USA) was used to perform all analyses. All analyses were stratified by sex.

\section{Results}

The median age at recruitment was 52.9 (range 35.7-65.8) years for men and 48.8 (range $35 \cdot 2-65 \cdot 6$ ) years for women. The baseline prevalence of supplement use in men and women was 28.5 and $38.6 \%$, respectively. After a median follow-up of 8.5 years, $14.6 \%$ of men and $22.9 \%$ of women were consistent supplement users, while $39.3 \%$ of men and $47.0 \%$ of women were inconsistent users, and $46.1 \%$ of men and $30.1 \%$ of women were never users (Table 1 ). In subjects who reported regular supplements at recruitment, 36.0\% of men and $26.6 \%$ of women stopped supplement use during follow-up. Meanwhile, in baseline non-users, $27.8 \%$ of men and $39.4 \%$ of women reported supplement use on later occasions.

After controlling for covariates, women were more likely to have consistent or inconsistent supplement use than men (Table 2 ). In both sexes, older age ( $\geq 50$ years) was significantly associated with increased likelihood of consistent or inconsistent supplement use. Subjects with higher educational level were more likely to have inconsistent supplement use, but only highly educated men were more likely to show consistent use. In women, being physically active was significantly associated with inconsistent or consistent supplement use (adjusted OR 1.35 (95\% CI 1.11, 1.64) for inconsistent use, and adjusted OR 1.28 (95\% CI 1.09, 1.51) for consistent use). Former smoking was also associated with inconsistent supplement use in women (adjusted OR 1.19; $95 \%$ CI 1.05, 1.35). Compared with those falling into the lowest tertile of lifetime alcohol intake, women in the middle and the highest tertile were more likely to show consistent and inconsistent supplement use. In both sexes, a lower BMI $\left(<25 \mathrm{~kg} / \mathrm{m}^{2}\right)$ was associated with higher likelihood of consistent or inconsistent supplement use. 
Table 1. Vitamin and/or mineral supplement use according to sex*

\begin{tabular}{|c|c|c|c|c|c|}
\hline & \multicolumn{2}{|c|}{ Men ( $n$ 8968) } & \multicolumn{2}{|c|}{ Women (n 10672) } & \multirow[b]{2}{*}{$P$} \\
\hline & $n$ & $\%$ & $n$ & $\%$ & \\
\hline \multicolumn{6}{|l|}{ Supplement use at baseline } \\
\hline Subjects with supplement use at baseline & 2552 & 28.5 & 4117 & 38.6 & \\
\hline Subjects without supplement use at baseline & 6416 & 71.5 & 6555 & $61 \cdot 4$ & $<0.0001$ \\
\hline \multicolumn{6}{|l|}{ Supplement-use categories } \\
\hline Consistent supplement users & 1313 & $14 \cdot 6$ & 2437 & $22 \cdot 9$ & \\
\hline Inconsistent supplement users & 3523 & $39 \cdot 3$ & 5019 & $47 \cdot 0$ & \\
\hline Never users & 4132 & $46 \cdot 1$ & 3216 & $30 \cdot 1$ & $<0.0001$ \\
\hline \multicolumn{6}{|l|}{ Subjects with supplement use at baseline } \\
\hline Subjects who kept using supplements & 1313 & 51.4 & 2437 & $59 \cdot 2$ & \\
\hline Subjects with interrupted use in FUP2 & 321 & $12 \cdot 6$ & 584 & $14 \cdot 2$ & \\
\hline Subjects who stopped using supplements & 918 & $36 \cdot 0$ & 1096 & $26 \cdot 6$ & $<0.0001$ \\
\hline \multicolumn{6}{|l|}{ Subjects without supplement use at baseline } \\
\hline Subjects who kept not using supplements & 4132 & 64.4 & 3216 & $49 \cdot 0$ & \\
\hline Subjects with interrupted non-use in FUP2 & 501 & $7 \cdot 8$ & 759 & 11.6 & \\
\hline Subjects who started supplement use & 1783 & $27 \cdot 8$ & 2580 & $39 \cdot 4$ & $<0.0001$ \\
\hline
\end{tabular}

Among self-reported health conditions at recruitment, hyperlipidaemia was significantly associated with increased odds of consistent and inconsistent supplement use in both sexes. Hypertension was only associated with inconsistent supplement use in men (adjusted OR 1.19; $95 \%$ CI 1.04, 1.36). Prevalent cancer at recruitment was associated with inconsistent supplement use in both men and women. However, only men were more likely to have consistent supplement use (adjusted OR 1.33; $95 \%$ CI 1.00, 1.76). Neither consistent nor inconsistent supplement use was found associated with baseline prevalent diabetes mellitus or history of myocardial infarction or stroke.

In both sexes, consistent, inconsistent and never supplement users had a significantly different intake of dairy products, fish, fruits and vegetables, and total meat (Table 3). Consistent supplement users had the highest intake of dairy products, fish, and fruits and vegetables but the lowest intake of total meat. The highest lifetime consumption of wine was also observed in consistent supplement users. Male consistent and inconsistent supplement users had a lower consumption of beer than never users, while female consistent and inconsistent supplement users had a higher consumption of spirits than never users. In women, consistent supplement users had a higher intake of total energy per $\mathrm{kg}$ body mass than inconsistent and never users.

\section{Discussion}

Vitamin and mineral supplement use is common in Germany. The German Nutrition Survey showed that $18.4 \%$ of men and $24.9 \%$ of women were regular vitamin or mineral supplement users $^{(12)}$. Using the same EPIC-Heidelberg cohort data, Reinert et al. reported that $33.5 \%$ of men and $40.1 \%$ of women were regular vitamin and/or mineral supplement users at recruitment $^{(13)}$. In our analysis that was limited to a subgroup of subjects who answered supplement-use questions in the baseline interactive interview and two subsequent follow-up surveys, we found that the baseline prevalence of regular supplement use was $28.5 \%$ in men and $38.6 \%$ in women, which was close to that of the entire cohort $(27.8 \%$ in men and $38.8 \%$ in women). The relatively higher prevalence of supplement use reported by Reinert et al. was obtained from the supplement-use information collected in the FFQ, which measured the regular use of any supplements in the previous 12 months, rather than in the previous 4 weeks. The lower prevalence reported in the German Nutrition Survey may be mainly due to its stricter definition of 'regular use' (supplementation of at least once per week in the previous year) and wider age range (18-79 years) of the study population. The present study showed in a prospective manner that only $14.6 \%$ of men and $22.9 \%$ of women were consistent supplement users, despite the high baseline prevalence of supplement use. One previous study specifically focusing on supplement use in women reported that $26.7 \%$ of the users became non-users while $21.8 \%$ of the non-users became users after a 2-year follow-up ${ }^{(14)}$. We found that among those who reported vitamin supplement use at recruitment, $36.0 \%$ of men and $26.6 \%$ of women discontinued their supplement use during follow-up, whereas in those baseline non-users, $27.8 \%$ of men and $39.4 \%$ of women started supplement use on later occasions. These findings suggest that supplement use is a fairly unstable behaviour in free-living individuals.

Studies have shown that women are more likely to use dietary supplements, in particular vitamin or mineral supplements $^{(1-3,5,6,15,16)}$. The present study further suggests that female supplement users are more likely to maintain supplement use in the long run than their male counterparts. Cross-sectional studies have suggested that increased age and educational level are associated with dietary supplement use $^{(1-3,5,6,15,16)}$. Our findings suggest that old individuals ( $\geq 50$ years) are more likely to have consistent supplement uses than young individuals. Higher educational level was associated with increased likelihood of inconsistent supplement use. However, a significant positive association between education level and consistent supplement use was observed only in men. Dietary supplement users have been reported having more physical activity than non-users ${ }^{(1,5,17)}$. In the present study, physical activity was measured by using the Cambridge Physical Activity Index, a combination 
Table 2. Association between vitamin and/or mineral supplement-use categories and baseline demographic, lifestyle and health characteristics ${ }^{\star}$ (Adjusted odds ratios and $95 \%$ confidence intervals)

\begin{tabular}{|c|c|c|c|c|c|c|c|c|c|c|c|c|c|c|}
\hline & \multicolumn{7}{|c|}{ Men ( $n$ 8968) } & \multicolumn{7}{|c|}{ Women (n 10 672) } \\
\hline & \multirow{2}{*}{$\frac{\mathrm{NU}}{n}$} & \multicolumn{3}{|c|}{ IU } & \multicolumn{3}{|c|}{$\mathrm{CU}$} & \multirow{2}{*}{$\frac{\mathrm{NU}}{n}$} & \multicolumn{3}{|c|}{ IU } & \multicolumn{3}{|c|}{$\mathrm{CU}$} \\
\hline & & $n$ & Adjusted OR† & $95 \% \mathrm{Cl}$ & $n$ & Adjusted OR† & $95 \% \mathrm{Cl}$ & & $n$ & Adjusted OR† & $95 \% \mathrm{Cl}$ & $n$ & Adjusted OR† & $95 \% \mathrm{Cl}$ \\
\hline Sex & 4132 & 3523 & 1.00 & & 1313 & 1.00 & & 3216 & 5019 & 2.91 & $2 \cdot 66,3 \cdot 19$ & 2437 & $2 \cdot 01$ & $1 \cdot 88,2 \cdot 16$ \\
\hline \multicolumn{15}{|l|}{ Baseline age (years) } \\
\hline$<50$ & 1862 & 1293 & 1.00 & & 344 & 1.00 & & 2028 & 2681 & 1.00 & & 922 & 1.00 & \\
\hline $50 \leq$ age $<60$ & 1645 & 1541 & $2 \cdot 07$ & $1 \cdot 78,2 \cdot 41$ & 642 & 1.31 & $1 \cdot 19,1.46$ & 840 & 1614 & 2.65 & $2 \cdot 33,3 \cdot 01$ & 1005 & 1.43 & $1.28,1.59$ \\
\hline$\geq 60$ & 625 & 689 & 2.84 & $2.35,3.42$ & 327 & 1.54 & $1.35,1.77$ & 348 & 724 & 3.54 & $2 \cdot 97,4.22$ & 510 & 1.61 & $1.38,1.88$ \\
\hline \multicolumn{15}{|l|}{ Education } \\
\hline None or primary school & 1315 & 1016 & 1.00 & & 295 & 1.00 & & 813 & 1280 & 1.00 & & 596 & 1.00 & \\
\hline Secondary schoolł & 1321 & 1159 & 1.45 & $1 \cdot 22,1 \cdot 72$ & 408 & $1 \cdot 17$ & $1.04,1.31$ & 1539 & 2488 & 1.21 & $1.06,1.39$ & 1254 & 1.06 & $0.94,1.18$ \\
\hline University & 1496 & 1348 & 2.02 & $1 \cdot 71,2 \cdot 39$ & 610 & 1.24 & $1.10,1.39$ & 864 & 1251 & $1 \cdot 10$ & $0.93,1.30$ & 587 & 0.97 & $0.85,1.11$ \\
\hline \multicolumn{15}{|l|}{ Physical activity } \\
\hline Inactive & 384 & 356 & 1.00 & & 151 & 1.00 & & 386 & 531 & 1.00 & & 286 & 1.00 & \\
\hline Moderately inactive & 1412 & 1174 & 0.90 & $0.72,1 \cdot 12$ & 452 & 0.94 & $0.80,1.11$ & 1154 & 1837 & $1 \cdot 11$ & $0.92,1.33$ & 855 & $1 \cdot 20$ & $1.03,1.40$ \\
\hline Moderately active & 1226 & 1052 & 0.91 & $0.73,1.15$ & 366 & 1.00 & $0.85,1.19$ & 943 & 1448 & $1 \cdot 17$ & $0.97,1.41$ & 701 & $1 \cdot 18$ & $1.01,1.38$ \\
\hline Active & 1110 & 941 & 1.02 & $0.81,1.29$ & 344 & 1.02 & $0.86,1.22$ & 733 & 1203 & 1.35 & $1 \cdot 11,1.64$ & 595 & 1.28 & $1.09,1.51$ \\
\hline \multicolumn{15}{|l|}{ Smoking status } \\
\hline Never smokers & 1318 & 1103 & 1.00 & & 430 & 1.00 & & 1561 & 2447 & 1.00 & & 1196 & 1.00 & \\
\hline Former smokers & 1878 & 1734 & $1 \cdot 11$ & $0.96,1.29$ & 674 & $1 \cdot 10$ & $0.99,1.22$ & 925 & 1496 & $1 \cdot 19$ & $1.05,1.35$ & 760 & 1.07 & $0.96,1.19$ \\
\hline Current smokers & 936 & 686 & 0.84 & $0.69,1.02$ & 209 & 0.94 & $0.83,1.08$ & 730 & 1076 & 1.05 & $0.91,1.22$ & 481 & 1.00 & $0.89,1.13$ \\
\hline \multicolumn{15}{|c|}{ Lifetime alcohol intake $(\mathrm{g} / \mathrm{d}) \S$} \\
\hline 1st tertile & 1385 & 1153 & 1.00 & & 445 & 1.00 & & 1172 & 1662 & 1.00 & & 728 & 1.00 & \\
\hline 2nd tertile & 1351 & 1218 & 0.95 & $0.81,1 \cdot 11$ & 429 & 1.05 & $0.94,1.18$ & 1028 & 1713 & 1.23 & $1.07,1.40$ & 833 & $1 \cdot 16$ & $1.04,1.29$ \\
\hline \multirow{2}{*}{\multicolumn{15}{|c|}{$\mathrm{BMI}\left(\mathrm{kg} / \mathrm{m}^{2}\right)$}} \\
\hline & & & & & & & & & & & & & & \\
\hline $\mathrm{BMI} \geq 30$ & 727 & 573 & 1.00 & & 184 & 1.00 & & 492 & 694 & 1.00 & & 307 & 1.00 & \\
\hline $25 \leq \mathrm{BMI}<30$ & 2108 & 1863 & 1.32 & $1.09,1.59$ & 680 & 1.15 & $1.01,1.31$ & 944 & 1495 & 1.24 & $1.04,1.48$ & 728 & $1 \cdot 13$ & $0.97,1.30$ \\
\hline $\mathrm{BMI}<25$ & 1297 & 1087 & 1.54 & $1.25,1.90$ & 449 & 1.15 & $0.99,1.33$ & 1780 & 2830 & 1.62 & $1.35,1.93$ & 1402 & 1.25 & $1.09,1.44$ \\
\hline \multicolumn{15}{|l|}{ Hypertension } \\
\hline No & 2746 & 2265 & 1.00 & & 801 & 1.00 & & 2493 & 3833 & 1.00 & & 1786 & 1.00 & \\
\hline Yes & 1386 & 1258 & $1 \cdot 19$ & $1.04,1.36$ & 512 & 1.04 & $0.94,1.15$ & 723 & 1186 & 1.03 & $0.90,1.18$ & 651 & 0.98 & $0.87,1.10$ \\
\hline \multicolumn{15}{|l|}{ Hyperlipidaemia } \\
\hline No & 2393 & 1899 & 1.00 & & 683 & 1.00 & & 2458 & 3465 & 1.00 & & 1565 & 1.00 & \\
\hline Yes & 1739 & 1624 & $1 \cdot 20$ & $1.06,1.37$ & 630 & $1 \cdot 14$ & $1.03,1.25$ & 758 & 1554 & 1.48 & $1.31,1.68$ & 872 & 1.37 & $1.23,1.52$ \\
\hline \multicolumn{15}{|l|}{ Diabetes mellitus } \\
\hline No & 3949 & 3323 & 1.00 & & 1232 & 1.00 & & 3144 & 4904 & 1.00 & & 2383 & 1.00 & \\
\hline Yes & 183 & 200 & 1.25 & $0.95,1.66$ & 81 & 1.21 & $0.98,1.49$ & 72 & 115 & 0.75 & $0.52,1.09$ & 54 & 0.92 & $0.68,1.26$ \\
\hline \multicolumn{15}{|c|}{ Myocardial infarction or stroke } \\
\hline No & 4005 & 3375 & 1.00 & & 1247 & 1.00 & & 3178 & 4958 & 1.00 & & 2395 & 1.00 & \\
\hline Yes & 127 & 148 & 1.26 & $0.92,1.72$ & 66 & $1 \cdot 18$ & $0.92,1.51$ & 38 & 61 & 1.04 & $0.66,1.64$ & 42 & 0.88 & $0.58,1.33$ \\
\hline \multicolumn{15}{|l|}{ Cancer } \\
\hline No & 4042 & 3415 & 1.00 & & 1256 & 1.00 & & 3124 & 4849 & 1.00 & & 2300 & 1.00 & \\
\hline Yes & 90 & 108 & 1.81 & $1 \cdot 28,2 \cdot 56$ & 57 & 1.33 & $1.00,1.76$ & 92 & 170 & 1.51 & $1.14,1.99$ & 137 & 1.05 & $0.81,1.36$ \\
\hline
\end{tabular}

$\mathrm{NU}$, never users; IU, inconsistent users; CU, consistent users.

${ }^{*}$ Multivariate multinomial logistic regression was performed, with never users as the reference category.

$\dagger$ All the covariates listed in the table were mutually adjusted for.

$¥$ Technical school and professional school were combined into this category.

$\S$ For men, the tertile cut-off points were 13.31 and $27.97 \mathrm{~g} / \mathrm{d}$. For women, the tertile cut-off points were 2.54 and $7.40 \mathrm{~g} / \mathrm{d}$. 
Table 3. Baseline intake of selected food groups, alcoholic beverages and total energy (Mean values and standard deviations)

\begin{tabular}{|c|c|c|c|c|c|c|c|}
\hline & \multicolumn{2}{|c|}{ NU } & \multicolumn{2}{|c|}{ IU } & \multicolumn{2}{|c|}{$\mathrm{CU}$} & \multirow[b]{2}{*}{ Omnibus $P$} \\
\hline & Mean & SD & Mean & SD & Mean & SD & \\
\hline \multicolumn{8}{|l|}{ Men } \\
\hline Subjects $(n)$ & \multicolumn{2}{|c|}{4132} & \multicolumn{2}{|c|}{3523} & \multicolumn{2}{|c|}{1313} & - \\
\hline Dairy products $(\mathrm{g} / \mathrm{d})$ & $220 \cdot 8$ & 219.4 & $244 \cdot 3^{*}$ & 250.2 & $267 \cdot 2^{*} \dagger$ & 255.5 & $<0.0001$ \\
\hline Fish $(g / d)$ & $21 \cdot 0$ & $20 \cdot 3$ & $22 \cdot 9^{*}$ & 24.4 & $25 \cdot 6^{*} \dagger$ & $24 \cdot 6$ & $<0.0001$ \\
\hline Cereals $(g / d)$ & 213.4 & 89.5 & $209 \cdot 0$ & $87 \cdot 2$ & 213.6 & $95 \cdot 7$ & 0.088 \\
\hline Fruits and vegetables $(\mathrm{g} / \mathrm{d})$ & $219 \cdot 7$ & $106 \cdot 3$ & $226 \cdot 4^{*}$ & 111.0 & $242 \cdot 4^{*} \dagger$ & 121.7 & $<0.0001$ \\
\hline Total meat $(\mathrm{g} / \mathrm{d})$ & $125 \cdot 6$ & 75.0 & $116 \cdot 3^{*}$ & 71.6 & $109 \cdot 7^{*} t$ & 68.9 & $<0.0001$ \\
\hline Wine $(g / d)$ & $115 \cdot 4$ & $176 \cdot 9$ & $128 \cdot 6^{\star}$ & $197 \cdot 8$ & $148 \cdot 3^{*} \dagger$ & 213.8 & $<0.0001$ \\
\hline Beer $(g / d)$ & $355 \cdot 8$ & 507.9 & $309 \cdot 2^{*}$ & 436.5 & $291.4 \dagger$ & 418.5 & $<0.0001$ \\
\hline Spirits $(\mathrm{g} / \mathrm{d})$ & 2.4 & $5 \cdot 8$ & $2 \cdot 8$ & 9.2 & $2 \cdot 8$ & $7 \cdot 6$ & 0.7212 \\
\hline Energy intake (kJ/d per kg) & $113 \cdot 6$ & 38.7 & $111 \cdot 3^{*}$ & 39.6 & $111 \cdot 7$ & $36 \cdot 6$ & 0.0071 \\
\hline \multicolumn{8}{|l|}{ Women } \\
\hline Subjects $(n)$ & \multicolumn{2}{|c|}{3216} & \multicolumn{2}{|c|}{5019} & \multicolumn{2}{|c|}{2437} & - \\
\hline Dairy products $(\mathrm{g} / \mathrm{d})$ & $254 \cdot 1$ & 209.5 & $254 \cdot 1^{*}$ & 209.5 & $286 \cdot 6^{*} \dagger$ & 234.4 & $<0.0001$ \\
\hline Fish $(g / d)$ & $15 \cdot 3$ & $15 \cdot 4$ & $16 \cdot 6^{*}$ & $17 \cdot 0$ & $18 \cdot 4^{*} \dagger$ & $19 \cdot 1$ & $<0.0001$ \\
\hline Cereals $(g / d)$ & $177 \cdot 0$ & $70 \cdot 6$ & $172 \cdot 8^{*}$ & 71.6 & $175 \cdot 9$ & $72 \cdot 7$ & 0.0071 \\
\hline Fruits and vegetables $(\mathrm{g} / \mathrm{d})$ & $251 \cdot 6$ & $130 \cdot 3$ & $256 \cdot 2$ & $121 \cdot 2$ & $274 \cdot 0^{*} \dagger$ & $130 \cdot 8$ & $<0.0001$ \\
\hline Total meat $(\mathrm{g} / \mathrm{d})$ & $80 \cdot 9$ & 53.4 & $75 \cdot 0^{*}$ & 47.9 & $70 \cdot 4^{*} \dagger$ & $48 \cdot 8$ & $<0.0001$ \\
\hline Wine $(g / d) \S$ & $82 \cdot 4$ & $130 \cdot 6$ & 84.7 & 128.4 & $97 \cdot 8^{*} \dagger$ & $145 \cdot 9$ & 0.0009 \\
\hline $\operatorname{Beer}(\mathrm{g} / \mathrm{d}) \S$ & 61.0 & $141 \cdot 3$ & 64.0 & 147.9 & $64 \cdot 2$ & $145 \cdot 8$ & 0.0723 \\
\hline Spirits $(g / d) \S$ & 0.7 & $2 \cdot 7$ & $0.8^{*}$ & 3.4 & $0.9 \dagger$ & 3.2 & 0.0152 \\
\hline Energy intake (kJ/d per kg) & $109 \cdot 9$ & $38 \cdot 7$ & 109.9 & 38.5 & $113 \cdot 0^{*} \dagger$ & $43 \cdot 1$ & 0.0358 \\
\hline
\end{tabular}

NU, never users; IU, inconsistent users; CU, consistent users.

${ }^{*}$ Mean value was significantly different from that of the NU group $(P<0.05)$

$\dagger$ Mean value was significantly different from that of the IU group $(P<0.05)$.

$\ddagger$ The Kruskal-Wallis test was used to perform overall comparison over the three groups, and the Bonferroni-Dunn test to conduct pairwise comparison when the omnibus difference was statistically significant.

$\S$ The amount of wine, beer and spirits represents the average daily consumption at recruitment.

of work activity and leisure-time activity. We found that physically active women were more likely to use supplements consistently. However, no significantly increased likelihood of supplement use was seen in physically active men. The possible explanation is that men who were categorised as physically active were more likely to do heavy manual work. These men tended to be younger and less educated, and were therefore less likely to use supplements.

Some studies have suggested that never smokers are more likely to use dietary supplements than former smokers or current smokers ${ }^{(1,3,18)}$. Nevertheless, Subar \& Block found that former smokers were the heaviest supplement users ${ }^{(19)}$. In addition, three studies in women reported higher dietary or vitamin and mineral supplement use in former smokers than in never smokers or current smokers ${ }^{(17,18,20)}$. We found that female former smokers were more likely to be inconsistent supplement users, suggesting that certain factors causing smoking cessation may also cause supplement use in this subgroup.

A study in French women has shown that dietary vitamin/ mineral supplement users drink less alcohol than non-users ${ }^{(14)}$, while a US study has observed that vitamin and mineral supplement use is more common in women who drink alcohol regularly ${ }^{(21)}$. In both studies, however, demographic and lifestyle covariates were not adjusted for. In other two studies, one has reported a non-significant inverse association between alcohol drinking and dietary supplementation after controlling for $\operatorname{covariates}^{(3)}$, whereas the other has shown that the frequency of wine or distilled spirit consumption is positively associated with multivitamin/mineral supplement use ${ }^{(22)}$.
One systematic review has shown that adults with a daily alcohol intake of $14.0 \mathrm{~g}$ have a reduced risk of $\mathrm{CHD}^{(23)}$. In the present study, we observed that women with an average lifetime alcohol intake of $12.9 \mathrm{~g} / \mathrm{d}$ (the median of the third tertile) were more likely to be consistent supplement users, suggesting a higher health consciousness in this group. Men in the 3rd tertile of lifetime alcohol intake were less likely to have consistent supplement use. The median of the lifetime alcohol intake in this group was $43.0 \mathrm{~g} / \mathrm{d}$, which was much greater than $20-24 \mathrm{~g} / \mathrm{d}$, the tolerable upper alcohol intake level recommended for adult German men ${ }^{(23)}$. This suggests a lower health consciousness in male heavy alcohol drinkers. We also found that the consumption of different types of alcoholic beverages varied across the three supplement-use categories (Table 3). In women, the highest consumption of wine was observed in consistent supplement users. In men, consistent supplement users consumed the highest amount of wine and the lowest amount of beer. Studies have shown that drinking more wine but less beer or spirits marks a higher socio-economic status ${ }^{(24,25)}$. Therefore, the present study suggests that consistent supplement use may be another marker of higher socio-economic status.

An inverse association of BMI and supplement use has been reported in cross-sectional studies ${ }^{(5,17,20)}$. In the present study, a lower BMI $\left(<25 \mathrm{~kg} / \mathrm{m}^{2}\right)$ was a predictor of consistent supplement use in both men and women. Only a few studies have examined the association between dietary supplement use and prevalent health conditions. Lyle et al. have reported that subjects with hypertension are less likely to use supplements than those without ${ }^{(2)}$. Two studies have reported 
that 69.3 and $74.0 \%$ of cancer survivors used dietary supplements (mainly multivitamins) after diagnosis ${ }^{(26,27)}$. In the present study, self-reported prevalent hypertension, hyperlipidaemia and cancer at recruitment were significantly associated with increased odds of inconsistent supplement use in men. All these health conditions except hypertension were also predictors of inconsistent supplement use in women. In both sexes, however, only hyperlipidaemia was a predictor of consistent supplement use, suggesting that vitamin and mineral supplements may be used for the purpose of controlling the blood lipid level.

It has been shown that supplement users consume more fruits, vegetables and dairy products than non-users ${ }^{(2,28,29)}$. In our cohort, consistent users consumed the highest amount of dairy products, fish, and fruits and vegetables but the lowest amount of total meat, suggesting that consistent supplement users have a healthier diet than inconsistent and never users. A study of Kim et al. has reported a significantly higher intake of total energy in female regular vitamin and mineral supplement users ${ }^{(21)}$. In the present study, male never users had the highest energy intake per $\mathrm{kg}$ body mass than inconsistent and consistent supplement users, even though the difference was only statistically significant between never users and inconsistent users. However, female consistent supplement users had a significantly higher energy intake per $\mathrm{kg}$ body mass than inconsistent and never supplement users. This might be because female consistent supplement users were more physically active.

All the previous studies examining the association between dietary supplement use and demographic, lifestyle and healthstatus factors were cross-sectionally designed. However, cross-sectional studies are problematic in investigating causal relationships. This drawback has been pointed out in some studies ${ }^{(3,6,7)}$. To the best of our knowledge, the present study is the first prospective cohort study focusing on the consistency of vitamin and mineral supplement use in free-living individuals. The aim of the present study was to predict subsequent consistent supplement use by using the baseline demographic, lifestyle and health-status characteristics as predictors. It is, however, necessary to point out that certain lifestyle factors, for example, physical activity, smoking status and BMI, may change over time. In addition, we used supplement-use information of the previous 4 weeks before each survey date, which apparently did not perfectly reflect the supplement-use status of the entire time period between two surveys. However, when we compared the supplementuse status of the previous 4 weeks obtained from the baseline interview with that of the previous year obtained from the FFQ, we found a good agreement, with a $\kappa$ coefficient of 0.898 for men and 0.876 for women, suggesting utilisation of the momentary assessment may not cause substantial bias. In the present study, we did not consider supplementation of specific vitamins and minerals, for the reason that $81.5 \%$ of supplement users took combinations of two or more different vitamins or minerals, from which we found it hard to obtain a dominant pattern. Lastly, we need to note that the prevalent health conditions at recruitment were self-reported, and hence the reporting bias was unable to be ruled out

In summary, the present study showed that vitamin and/or mineral supplement use was a fairly unstable behaviour in free-living individuals. Being a woman and of older age
( $\geq 50$ years), having a lower BMI $\left(<25 \mathrm{~kg} / \mathrm{m}^{2}\right)$ and self-reported hyperlipidaemia were predictors of consistent supplement use. Higher educational level was an additional predictor of consistent supplement use for men, while physical activeness and higher lifetime alcohol consumption were additional predictors for women. Consistent users had the highest intake of dairy products, fish, fruits and vegetables, and wine but the lowest intake of total meat. We conclude that consistent users have a cluster of favourable lifestyle and dietary factors that need to be adjusted for when investigating the health effects of dietary supplementation on chronic diseases, particularly cancer and CVD. Furthermore, more efficient instruments need to be developed for the accurate measurement of long-term consistent supplement use given the changeability of this behaviour in real life.

\section{Acknowledgements}

The present study was supported by the Deutsche Krebshilfe (grant no. 70-488-Ha I), the Deutsche Forschungsgemeinschaft and the Graduiertenkolleg 793. We thank all the subjects of the present study for their continued participation.

K. L. conducted the statistical analyses and drafted the manuscript. S. R. provided instructions on statistical methods. S. R., J. L. and R. K. participated in results interpretation and manuscript review.

We are also thankful to Ilona Krüger-Friedemann, Jutta Kneisel and Jutta Schmitt for coding and preparing the dataset.

The authors declare no conflict of interest.

\section{References}

1. Ishihara J, Sobue T, Yamamoto S, et al. (2003) Demographics, lifestyles, health characteristics, and dietary intake among dietary supplement users in Japan. Int J Epidemiol 32, 546-553.

2. Lyle BJ, Mares-Perlman JA, Klein BE, et al. (1998) Supplement users differ from nonusers in demographic, lifestyle, dietary and health characteristics. $J$ Nutr 128, 2355-2362.

3. McNaughton SA, Mishra GD, Paul AA, et al. (2005) Supplement use is associated with health status and healthrelated behaviors in the 1946 British birth cohort. J Nutr 135, 1782-1789.

4. Patterson RE, Neuhouser ML, White E, et al. (1998) Cancerrelated behavior of vitamin supplement users. Cancer Epidemiol Biomarkers Prev 7, 79-81.

5. Rock CL (2007) Multivitamin-multimineral supplements: who uses them? Am J Clin Nutr 85, 277S-279S.

6. Satia-Abouta J, Kristal AR, Patterson RE, et al. (2003) Dietary supplement use and medical conditions: the VITAL study. Am J Prev Med 24, 43-51.

7. Block G, Jensen CD, Norkus EP, et al. (2007) Usage patterns, health, and nutritional status of long-term multiple dietary supplement users: a cross-sectional study. Nutr J 6, 30.

8. Patterson RE, Neuhouser ML, Hedderson MM, et al. (2003) Changes in diet, physical activity, and supplement use among adults diagnosed with cancer. $J$ Am Diet Assoc 103, 323-328.

9. Riboli E (2001) The European Prospective Investigation into Cancer and Nutrition (EPIC): plans and progress. J Nutr 131, 170S-175S.

10. Boeing H, Korfmann A \& Bergmann MM (1999) Recruitment procedures of EPIC-Germany. European Investigation into Cancer and Nutrition. Ann Nutr Metab 43, 205-215. 
11. Wareham NJ, Jakes RW, Rennie KL, et al. (2003) Validity and repeatability of a simple index derived from the Short Physical Activity Questionnaire used in the European Prospective Investigation into Cancer and Nutrition (EPIC) study. Public Health Nutr 6, 407-413.

12. Beitz R, Mensink GB, Fischer B, et al. (2002) Vitamins - dietary intake and intake from dietary supplements in Germany. Eur J Clin Nutr 56, 539-545.

13. Reinert A, Rohrmann S, Becker N, et al. (2007) Lifestyle and diet in people using dietary supplements: a German cohort study. Eur J Nutr 46, 165-173.

14. Touvier M, Kesse E, Volatier JL, et al. (2006) Dietary and cancer-related behaviors of vitamin/mineral dietary supplement users in a large cohort of French women. Eur J Nutr 45, 205-214.

15. Balluz LS, Okoro CA, Bowman BA, et al. (2005) Vitamin or supplement use among adults, behavioral risk factor surveillance system, 13 states, 2001. Public Health Rep 120, $117-123$.

16. Schwarzpaul S, Strassburg A, Luhrmann PM, et al. (2006) Intake of vitamin and mineral supplements in an elderly German population. Ann Nutr Metab 50, 155-162.

17. Yu SM, Kogan MD \& Huang ZJ (2003) Vitamin-mineral supplement use among US women, 2000. J Am Med Womens Assoc 58, $157-164$.

18. Kirk SF, Cade JE, Barrett JH, et al. (1999) Diet and lifestyle characteristics associated with dietary supplement use in women. Public Health Nutr 2, 69-73.

19. Subar AF \& Block G (1990) Use of vitamin and mineral supplements: demographics and amounts of nutrients consumed. The 1987 Health Interview Survey. Am J Epidemiol 132, 1091-1101.
20. Knudsen VK, Rasmussen LB, Haraldsdottir J, et al. (2002) Use of dietary supplements in Denmark is associated with health and former smoking. Public Health Nutr 5, 463-468.

21. Kim I, Williamson DF, Byers T, et al. (1993) Vitamin and mineral supplement use and mortality in a US cohort. Am J Public Health 83, 546-550.

22. Radimer K, Bindewald B, Hughes J, et al. (2004) Dietary supplement use by US adults: data from the National Health and Nutrition Examination Survey, 1999-2000. Am J Epidemiol 160, 339-349.

23. Burger M, Brönstrup A \& Pietrzik K (2004) Derivation of tolerable upper alcohol intake levels in Germany: a systematic review of risks and benefits of moderate alcohol consumption. Prev Med 39, 111-127.

24. Marques-Vidal P, Arveiler D, Evans A, et al. (2000) Patterns of alcohol consumption in middle-aged men from France and Northern Ireland. The PRIME study. Eur J Clin Nutr 54, 321-328.

25. Nielsen NR, Schnohr P, Jensen G, et al. (2004) Is the relationship between type of alcohol and mortality influenced by socioeconomic status? J Intern Med 255, 280-288.

26. Ferrucci LM, McCorkle R, Smith T, et al. (2009) Factors related to the use of dietary supplements by cancer survivors. J Altern Complement Med 15, 673-680.

27. Miller P, Demark-Wahnefried W, Snyder DC, et al. (2008) Dietary supplement use among elderly, long-term cancer survivors. J Cancer Surviv 2, 138-148.

28. Frank E, Bendich A \& Denniston M (2000) Use of vitaminmineral supplements by female physicians in the United States. Am J Clin Nutr 72, 969-975.

29. Harrison RA, Holt D, Pattison DJ, et al. (2004) Are those in need taking dietary supplements? A survey of 21923 adults. Br J Nutr 91, 617-623. 